\title{
Existence and Uniqueness of Elliptic Periodic Solutions of the Brillouin Electron Beam Focusing System
}

\author{
Pedro J. Torres*, ${ }^{*}$ \\ Departamento de Matemática Aplicada, Universidad de Granada, 18071 Granada, Spain \\ Communicated by B. Straughan
}

We find conditions for the existence of an elliptic periodic solution of a singular equation that governs the motion of a magnetically focused axially symmetric electron beam with Brillouin flow by using a monotone iterative scheme starting from a couple of upper and lower solutions on the reversed order. Also, a uniqueness result is proved by using Brouwer degree and index of solutions. Copyright (C) 2000 John Wiley \& Sons, Ltd.

This note concerns the existence of positive $2 \pi$-periodic solutions of the equation

$$
x^{\prime \prime}(t)+a(1+\cos (t)) x(t)=\frac{b}{x(t)}
$$

where $a, b$ are positive constants.

The physical meaning of this equation arises in the context of Electronics, since it governs the motion of a magnetically focused axially symmetric electron beam under the influence of a Brillouin flow, as shown in [1]. From the mathematical point of view, equation (1) is a singular perturbation of a Mathieu equation.

Several papers have studied the existence of periodic solutions of (1). It seems that the first reference in this line is [3], where the existence of periodic solutions is proved when $a \leqslant \frac{1}{16}$ by using an analysis of the phase plane. Afterwards, this result was improved in [8] and more recently in [9] by using coincidence degree. However, to the best of my knowledge, nothing has been published concerning the uniqueness or stability of such a solution. In this direction, two results are presented here, the first of them is the following.

Theorem 1. If $a \leqslant \frac{1}{16}$, equation (1) has a $2 \pi$-periodic solution, which is elliptic.

As is known, a periodic solution is said to be elliptic if the linearized equation is elliptic, that is, if the Floquet multipliers have modulus 1 and are different from \pm 1 .

\footnotetext{
* Correspondence to: P. J. Torres, Departamento de Matemática Aplicada, Universidad de Granada, 18071 Granada, Spain

${ }^{\dagger}$ Supported by D.G.E.S. PB95-1203, M.E.C., Spain
} 
This implies that the linearized equation is stable, although Lyapunov stability of the solution is not assured since it may depend on the non-linear terms. Therefore, the stability of such a solution remains an interesting open problem that should be tackled by other techniques like the theory of Birkhoff normal forms. In this sense, results of this paper can be seen as a first step in this direction.

Note that we obtain the same estimate of Ding with additional information about the characteristic multipliers. The proof of existence is based on a monotone iterative scheme starting from upper and lower solutions on the reversed order. Meanwhile, the elliptic character is proved from the basic properties of Floquet multipliers. At the end of this paper a uniqueness result is proved by using topological degree and index.

We begin by recalling the classical definition of upper and lower solutions. Let us consider the periodic problem

$$
\begin{aligned}
& x^{\prime \prime}+f(t, x(t))=0 \\
& x(0)=x(2 \pi), x^{\prime}(0)=x^{\prime}(2 \pi)
\end{aligned}
$$

with $f$ being continuous.

Definition 1. A function $\alpha \in C^{2}([0,2 \pi])$ is said to be a lower solution of problem (2) if and only if

(i) for all $t \in[0,2 \pi], \alpha^{\prime \prime}(t)+f(t, \alpha(t)) \geqslant 0$,

(ii) $\alpha(0)=\alpha(2 \pi), \alpha^{\prime}(0) \geqslant \alpha^{\prime}(2 \pi)$.

In the same way, an upper solution $\beta(t)$ is defined by reversing the respective inequalities in the previous definition. A lower solution (resp. upper solution) is called strict if the inequality in (i) is strict.

The next result provides a classical monotone iterative scheme. The proof relies basically on the Antimaximum Principle and can be found for instance in [2] or [4].

Theorem 2. Let $\alpha, \beta \in C^{2}([0,2 \pi])$ be a couple of lower and upper solutions such that $\beta(t) \leqslant \alpha(t)$ for all $t \in[0,2 \pi]$. Assume moreover that for any $t \in[0,2 \pi]$ and $u, v$ with $\beta(t) \leqslant u \leqslant v \leqslant \alpha(t)$, we have

$$
\frac{1}{4} u-f(t, u) \leqslant \frac{1}{4} v-f(t, v)
$$

Then, there exist two monotone sequences $\left\{\alpha_{n}\right\}_{n},\left\{\beta_{n}\right\}_{n}$ such that

$$
\alpha=\alpha_{0} \geqslant \cdots \geqslant \alpha_{n-1} \geqslant \alpha_{n} \geqslant \cdots \geqslant \beta_{n} \geqslant \beta_{n-1} \geqslant \cdots \geqslant \beta_{0}=\beta
$$

which converge uniformly to extremal solutions of problem (2) in $[\beta, \alpha]$. Also, if $\alpha$ and $\beta$ are strict lower and upper solutions, the previous inequalities are strict.

Now, we prove a result along the lines of Lemma 1.4 in [5].

Lemma 1. Let $w(t)$ be a non-constant continuous $2 \pi$-periodic function such that $w(t) \leqslant \frac{1}{4}$ for all $t$. Then, Hill's equation $y^{\prime \prime}+w(t) y=0$ does not admit negative Floquet 
multipliers. If moreover $w(t)>0$ for all $t$, then Hill's equation does not admit real Floquet multipliers.

Proof. An elementary application of Sturm comparison theory shows that if $t_{1}<t_{2}$ are consecutive zeros of a solution of Hill's equation, then $t_{2}-t_{1}>2 \pi$. Now, if $\lambda<0$ is a negative Floquet multiplier, there exists a non-trivial solution such that $y(t+2 \pi)=\lambda y(t)$ for all $t$, but this implies that $y\left(t_{0}\right)=y\left(t_{0}+2 \pi\right)=0$ for some $t_{0} \in[0,2 \pi]$, a contradiction.

Now assume that $w(t)>0$ for all $t$. It is known that if $\lambda_{1}, \lambda_{2}$ are the Floquet multipliers, then $\lambda_{1} \lambda_{2}=1$. First suppose that $\lambda_{1}=1$. This implies that Hill's equation has a non-trivial $2 \pi$-periodic solution. Then, such a solution must have a constant sign since the distance between two consecutive zeros is greater than $2 \pi$, and a contradiction is obtained by integrating the equation over a period. The remaining case is that $0<\lambda_{1}<1<\lambda_{2}$. Then, there exists a solution $y(t)$ such that $y(t+2 \pi)=\lambda_{1} y(t)$ for all $t$, and it must have a constant sign by the same consideration as before. If we suppose for instance that $y(t)$ is positive, $y^{\prime \prime}(t)=-w(t) y(t)<0$ for all $t$, so $y(t)$ is concave. On the other hand, $y(t+2 n \pi)=\lambda_{1}^{n} y(t)$ for all $t$, in consequence $\lim _{t \rightarrow \infty} y(t)=0$, contradicting the fact that $y$ is concave and positive.

Proof of Theorem 1. It is easy to verify that $\beta(t):=\sqrt{(b / 2 a)}$ is a constant upper solution of equation (1). Also, it is not hard to prove that there exists $K^{*}>0$ such that $\alpha(t):=K+\cos (t)$ is a lower solution for every $K>K^{*}$. If moreover $K>1+\sqrt{(b / 2 a)}$, then we have a couple of lower and upper solutions such that $\beta(t) \leqslant \alpha(t)$ for all $t \in[0,2 \pi]$. Defining $f(t, x):=a(1+\cos (t)) x-b / x$, assumption (3) of Theorem 2 is easily verified since $f_{x}(t, x) \leqslant \frac{1}{4}$ for all $x \geqslant \beta(t)$, whence $\frac{1}{4} x-f(t, x)$ is strictly increasing. In a consequence, there exists a $2 \pi$-periodic solution $x(t)$ between $\beta$ and $\alpha$.

Also, the linearized equation for $x(t)$ is

$$
y^{\prime \prime}+f_{x}(t, x(t)) y=0
$$

and $0<f_{x}(t, x) \leqslant \frac{1}{4}$, so $x(t)$ is elliptic as a direct consequence of Lemma 1 .

Finally, we conclude with a result about uniqueness by using the relationship between the index of a periodic solution and the Floquet multipliers of the linearized equation. The index of a periodic solution is defined as the Brouwer degree of the identity minus the Poincaré map $P$ in a small ball (see for instance [7] for details).

Theorem 3. If $a \leqslant \frac{1}{16}$, equation (1) has a unique $2 \pi$-periodic solution which satisfies $x(t)>\sqrt{(b / 2 a)}$ for all $t$.

Proof. As existence is shown by Theorem 1, we only have to prove uniqueness. The proof is divided into three steps.

- Every periodic solution such that $x(t)>\sqrt{(b / 2 a)}$ for all $t$ is elliptic. It is a direct consequence of Lemma 1. 
- Let $\beta(t), \alpha(t)$ be the upper and lower solutions on the reversed order defined as in the proof of Theorem 1 , and define the set $\Omega:=\left[x \in C^{2}(\mathbb{R} / 2 \pi \mathbb{Z}): \beta(t)<x(t)<\alpha(t)\right.$, $\left.\left|x^{\prime}(t)\right|<R\right\}$ where $R$ is a given constant only depending on $\alpha, \beta$. Then

$$
\operatorname{deg}(I-P, \Omega)=1
$$

The proof is essentially the same as that of Lemma 3.2 in [6], where the reasoning is done when lower and upper solutions are in the 'right' order. Let us define the functions

$$
\delta(\beta, x, \alpha):= \begin{cases}\beta(t) & \text { if } x(t) \leqslant \beta(t) \\ x(t) & \text { if } \beta(t) \leqslant x(t)<\alpha(t) \\ \alpha(t) & \text { if } \alpha(t) \leqslant x(t)\end{cases}
$$

and

$$
F(t, x):=f(t, \delta(\beta, x, \alpha))+\frac{1}{4}(x-\delta(\beta, x, \alpha))
$$

Considering the modified equation

$$
x^{\prime \prime}+F(t, x)=0
$$

the Antimaximum Principle implies that every periodic solution lies between $\beta$ and $\alpha$ and is indeed a solution of (2). Now, if we consider the homotopy

$$
x^{\prime \prime}+\frac{1}{4} x+\lambda\left[F(t, x)-\frac{1}{4} x\right]=0, \quad \lambda \in[0,1]
$$

it is not hard to find a priori bounds for the $2 \pi$-periodic solutions of (5). This can be proved if we consider equation (5) as a linear complete equation $x^{\prime \prime}+\frac{1}{4} x+p_{\lambda}(t)=0$, where $p_{\lambda}(t)=\lambda f(t, \delta(\beta, x, \alpha))-\frac{1}{4} \delta(\beta, x, \alpha)$. Clearly, $p_{\lambda}(t)$ is bounded by a constant which does not depend on $\lambda$. As the homogeneous part has no $2 \pi$-periodic solutions, then the unique $2 \pi$-periodic solution of the complete equation is

$$
x(t)=\int_{0}^{t} G(t, s) p_{\lambda}(s) \mathrm{d} s
$$

where $G(t, s)$ is the Green's function associated with the periodic problem. As $G(t, s)$ is obviously bounded and does not depend on $\lambda$, our assertion follows after some easy computations.

An easy computation proves that when $\lambda=0$ the Brouwer degree is 1 , so we finish taking into account the invariance of topological degree by homotopies.

- Let us define $\alpha_{K}(t):=K+\cos (t)$. Then, the number of periodic solutions between $\sqrt{(b / 2 a)}$ and $\alpha_{K}$ is finite. We know from the first step that every solution $x(t)>\sqrt{(b / 2 a)}$ is non-degenerate (that is, 1 is not a characteristic multiplier), but this implies that it is isolated, which means that the corresponding initial condition is an isolated fixed point of the Poincare map. Now, we finish taking into account that the number of isolated points on a bounded domain is always finite. 
- Conclusion: From basic properties of the degree it is known that if the number of solutions on a set is finite, then the Brouwer degree on a set is the sum of the respective indexes of the solutions on this set. But it is known that the index of an elliptic solution is 1 . Therefore, the degree is 1 and the index of every solution with lower bound $\sqrt{(b / 2 a)}$ is also 1 , so there is at most one solution between $\sqrt{(b / 2 a)}$ and $\alpha_{K}$. As $K$ can be chosen as large as we wish, the proof is finished.

As a final remark, we emphasize that the problem of uniqueness not involving lower bounds remains open.

\section{Acknowledgement}

I wish to thank Rafael Ortega for some useful comments and key ideas about this topic.

\section{References}

1. Bevc V, Palmer JL, Süsskind C. On the design of the transition region of axi-symmetric magnetically focusing beam values. J. British Inst. Radio Eng. 1958; 18: 696-708.

2. De Coster C, Habets P. Upper and lower solutions in the theory of ODE boundary value problems: classical and recent results. In Nonlinear Analysis and Boundary Value Problems for Ordinary Differential Equations, Zanolin F, (ed). CISM-ICMS Courses and Lectures, vol. 371. Springer: New York, 1996.

3. Ding T. A boundary value problem for the periodic Brillouin focusing system. Acta Sci. Natur. Univ. Pekinensis 1965; 11: 31-38 (in Chinese).

4. Omari P, Trombetta M. Remarks on the lower and upper solutions method for second- and third-order periodic boundary value problems. Appl. Math. Comput. 1992; 50: 1-21.

5. Ortega R. Stability and index of periodic solutions of an equation of Duffing type. Bolletino UMI 1989; 7: 533-546.

6. Ortega R. Topological degree and stability of periodic solutions for certain differential equations. J. London Math. Soc. 1990; 42: 505-516.

7. Ortega R. Some applications of the topological degree to stability theory. In Topological Methods in Differential Equations and Inclusions, N.A.T.O. ASI Series 6, Kluwer Academic Publishers: Dordrecht, 1994.

8. Ye Y, Wang X. Nonlinear differential equations in electron beam focusing theory. Acta Math. Appl. Sinica 1978; 1: 13-41 (in Chinese).

9. Zhang M. Periodic solutions of Liénard equations with singular forces of repulsive type. J. Math. Anal. Appl. 1996; 203: 254-269. 